EPiC Series in Engineering
Volume 3, 2018, Pages 290-294
HIC 2018. 13th International
Conference on Hydroinformatics

\title{
Performance of Global Atmospheric Datasets towards Groundwater Management
}

\author{
P. Bongioannini Cerlini ${ }^{1}$, S. Meniconi ${ }^{2}$, and B. Brunone ${ }^{3}$ \\ ${ }^{1}$ CIRIAF/Centre for Climate and Climate Change (CRC), University of Perugia, 06125 Perugia, \\ Italy \\ paolina.cerlini@unipg.it \\ 2 Department of Civil and Environmental Engineering, University of Perugia, 06125 Perugia, Italy \\ silvia.meniconi@unipg.it \\ ${ }^{3}$ Department of Civil and Environmental Engineering, University of Perugia, 06125 Perugia, Italy \\ bruno. brunone@unipg.it
}

\begin{abstract}
With the aim of refining a reliable tool for groundwater management, the ERA-Interim and ERA5 global atmospheric datasets provided by the European Centre for MediumRange Weather Forecasts (ECMWF) are examined. Attention is focused on the analysis of the behavior of the soil moisture content. The performance of ERA-Interim and ERA5 is evaluated by considering the water table measurements at three sites in the Umbria region as well as the dynamics of water flow towards the groundwater.
\end{abstract}

\section{Introduction}

Climate change influences the hydrological cycle with direct effects on groundwater resources, one of the most important supply source for human consumption and irrigation. In the framework of assessing water balance and the related hydraulic works for water supply, it is crucial to correlate quantitatively climate trends, precipitation and groundwater behavior. In a previous paper [2], data from ERA-Interim reanalysis of meteorological observations produced by the European Centre for Medium-Range Weather Forecasts (ECMWF) have been compared to the local water table measurements given by the monitoring network managed by the Regional Agency for Environmental Protection of the Umbria Region (ARPA Umbria, Italy). The pointed out good correlation between the trend of the soil moisture (i.e., the result of precipitation over all meteorological space-time scales) and local water table data of unconfined aquifers authorizes further in-depth analyses. Specifically, the ERA5 reanalysis, the most updated ECMWF reanalysis (http://apps.ecmwf.int/data-catalogues/era5) are considered. One of the improvements of ERA5 with respect to ERA-Interim is the inclusion of the more refined H-TESSELL hydrologic scheme, which is based on a revised formulation of the soil hydrological conductivity and diffusivity. This makes ERA5 very attractive with regard to the analysis of groundwater resources. In this paper meteorological observations from ERA-INTERIM and ERA5 reanalyses are compared with the aim of evaluating the effect of: i) the different models and procedures used to obtain atmospheric global datasets, and ii) the characteristics of 


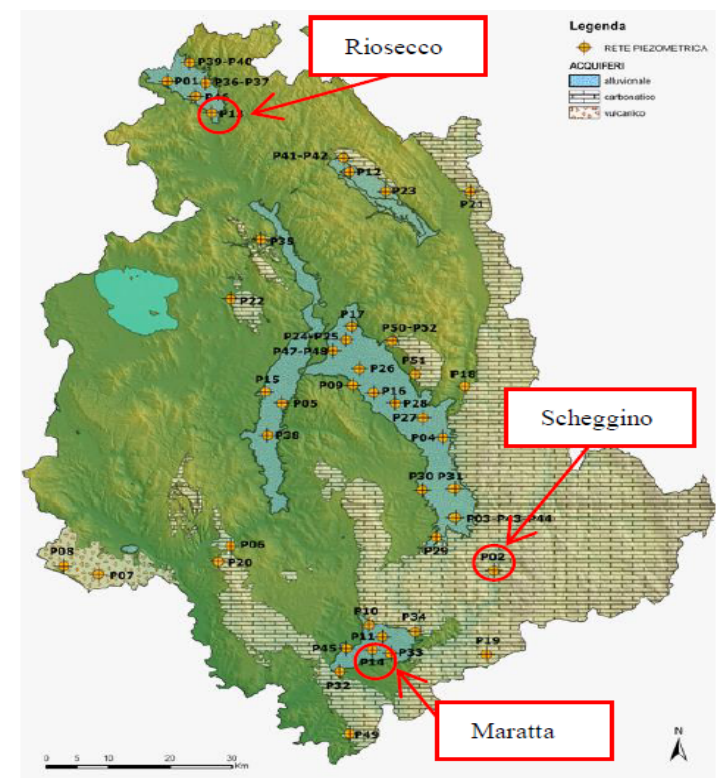

Figure 1: Water table monitoring network managed by ARPA Umbria (in red the sites considered in this study).

data on which reanalyses are based (e.g., their temporal and spatial resolution). Attention is focused on the behavior of the water flow towards water table as a preliminary phase of the water budget assessment. The ultimate goal of this research is to check whether the global atmospheric datasets - specifically the behavior of the soil moisture volumetric content may allow estimating the available groundwater resources at a regional scale.

\section{Materials and Methods}

Two kinds of data have been used for evaluating the behavior of groundwater: the soil moisture content, $\theta$, provided by the ERA-INTERIM and ERA5 reanalysis datasets of ECMWF at four different depths, $z$, from the ground level (layer $1: z=0-7 \mathrm{~cm}$; layer $2: z=7-28 \mathrm{~cm}$; layer 3 : $z=28-100 \mathrm{~cm}$; and layer $4: z=100-289 \mathrm{~cm}$, respectively); and the water table elevation, $h$, given by ARPA Umbria (Italy). ARPA Umbria has been collecting water table data at 52 sites in the region since early 2000s (Fig. 1); for each of these sites, the value of $h$ is provided as the mean daily value [4]. In this study, attention is focused on the three sites (Riosecco, Scheggino, and Maratta) spread throughout the Umbria region [4] (Fig. 1).

\section{ECMWF soil moisture data: ERA-INTERIM vs. ERA5}

The improved modeling of the atmospheric processes and soil characteristics in ERA5 reflects clearly in a more credible dynamics of the soil moisture behavior with more valuable differences between the value of $\theta$ at different depths in ERA5 with respect to ERA-INTERIM (Fig. 2). One of the effects of the much larger spatial resolution of ERA5 data can be noticed in Fig.3 

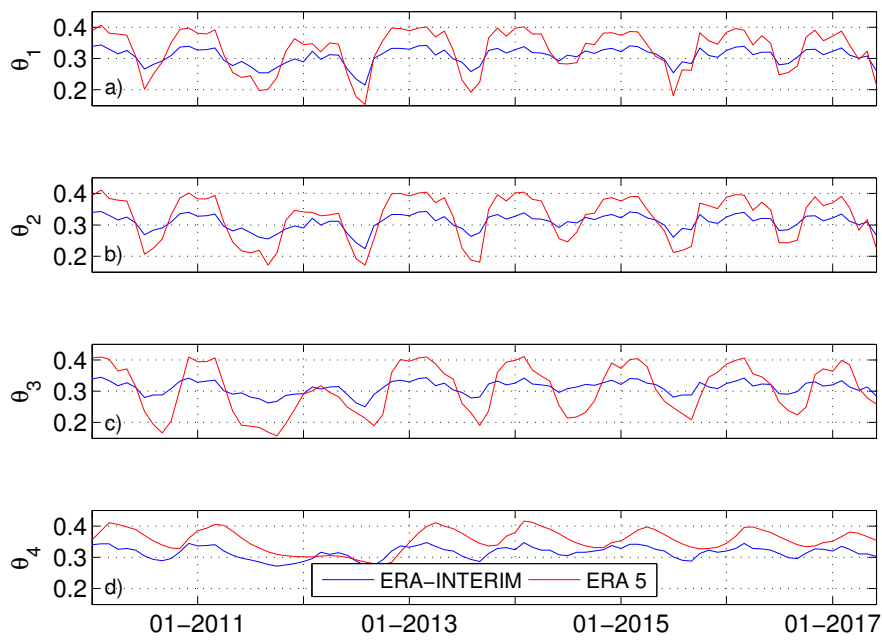

Figure 2: Riosecco site: ERA-INTERIM vs. ERA5 - time series of the monthly-daily means of the volumetric soil water content, $\theta\left(\mathrm{m}^{3} / \mathrm{m}^{3}\right)$, at layer: a) $1(z=0-7 \mathrm{~cm})$; b) $2(z=7-28 \mathrm{~cm})$; c) $3(z=28-100 \mathrm{~cm})$; and d) $4(z=100-289 \mathrm{~cm})$.

where values at the selected sites from ERA-INTERIM do not exhibit any differences. On the contrary, in principle ERA5 data show admissible differences and make more reliable the related areal average values of Fig. 4.

\section{Concluding Remarks}

Numerical experiments based on reanalysis data and comparisons with measured water table elevations point out the improvements of ERA5 with respect to ERA-INTERIM. Specifically, ERA5 captures more reliably the soil moisture behavior. However, a further check is needed with regard to its consistency.

As a first taste of a possible use of the global atmospheric datasets as a reliable tool for water resources management, water flow towards water table, $F_{w}$, has been evaluated by means of the Richards equation:

$$
F_{w}=k\left(\frac{\psi_{i+1}-\psi_{i}}{\Delta z}-1\right)
$$

by assuming free drainage as bottom boundary condition [1]; in Eq. (1), the hydraulic conductivity, $k$, assumed as spatially variable according to the global soil texture map reported in [3], is given by the van Genuchten equation [5], and the pressure head, $\psi$, has been evaluated by assuming $\theta=\theta_{4}$. As a reference, in Figs. 5a) and 5b) the time-history of $F_{w}$ for Riosecco site is compared with the relevant water table measurements. As pointed out in Fig. 5c), a yearly periodicity can be observed as well as a one or two months time shift between the maximum values of $F_{w}$ and $h$. 


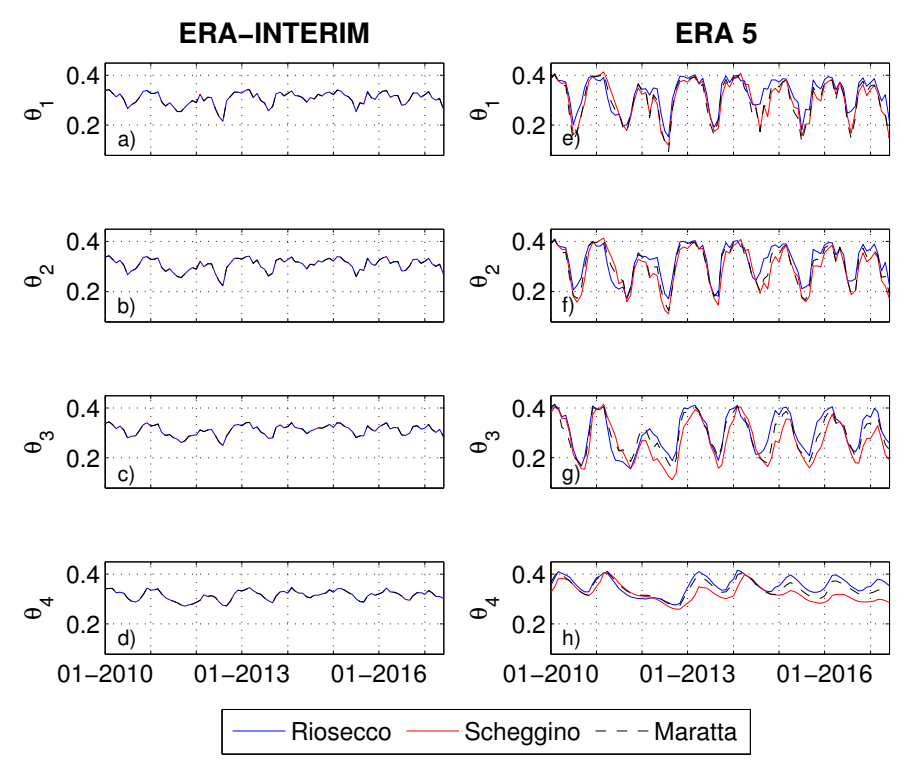

Figure 3: Comparison between the three different sites considered in this study: ERA-INTERIM vs. ERA 5 - time series of the monthly-daily means of the volumetric soil water content, $\theta$ $\left(\mathrm{m}^{3} / \mathrm{m}^{3}\right)$, at layer: a), e) $\left.\left.\left.\left.1(z=0-7 \mathrm{~cm}) ; \mathrm{b}\right), \mathrm{f}\right) 2(z=7-28 \mathrm{~cm}) ; \mathrm{c}\right), \mathrm{g}\right) 3(z=28-100 \mathrm{~cm})$; and d), h) $4(z=100-289 \mathrm{~cm})$.
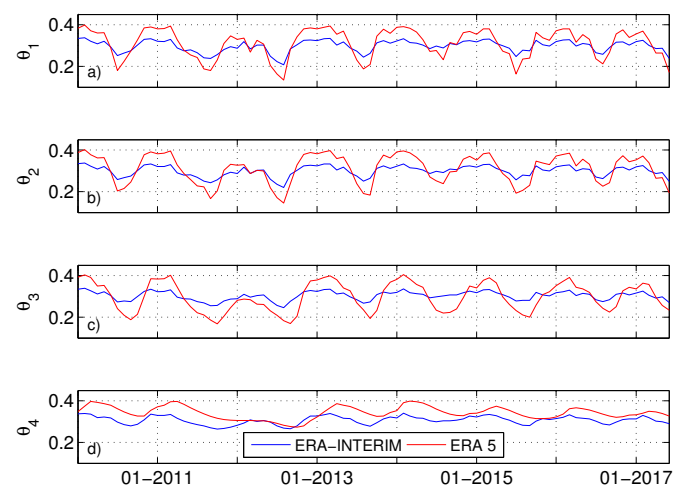

Figure 4: ERA-INTERIM vs. ERA5 - time series of Umbria areal average of the monthly-daily means of the volumetric soil water content, $\theta\left(\mathrm{m}^{3} / \mathrm{m}^{3}\right)$, at layer: a) $1(z=0-7 \mathrm{~cm})$; b) $2(z=$ $7-28 \mathrm{~cm})$; c) $3(z=28-100 \mathrm{~cm})$; and d) $4(z=100-289 \mathrm{~cm})$. 

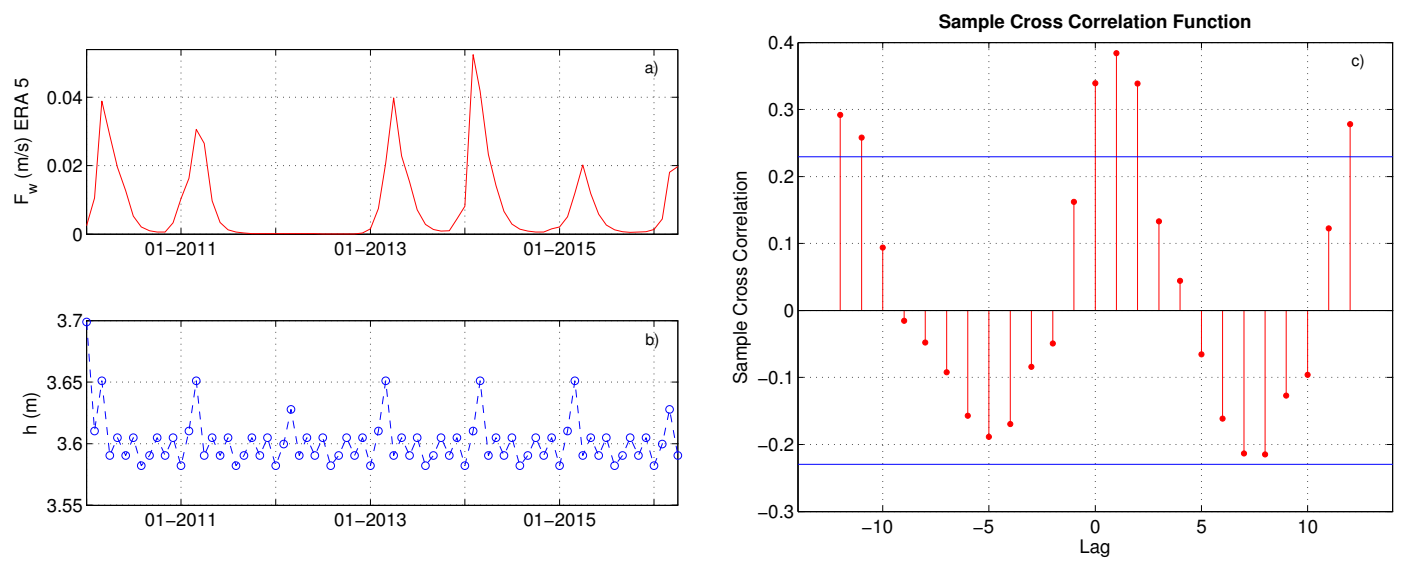

Figure 5: Riosecco site: monthly-daily mean time series of the: a) water flow from layer $4(z=$ $100-289 \mathrm{~cm})$ to the aquifer, $F_{w}$; b) water table elevation, $h$; c) sample cross correlation between $F_{w}$ and $h$.

\section{Acknowledgments}

Support from Italian MIUR and University of Perugia is acknowledged within the program Dipartimenti di Eccellenza 2018-2022. The support of M. Nucci of ARPA Umbria for the water table measurements is highly appreciated.

\section{References}

[1] G. Balsamo, P. Viterbo, A. Beljaars, B. van den Hurk, M. Hirschi, A.K. Betts, and K. Scipal. A revised hydrology for the ECMWF model: verification from field site to terrestrial water storage and impact in the integrated forecast system. J. Hydrometeorol., 10:623-643, 2009.

[2] P. Bongioannini Cerlini, S. Meniconi, and B. Brunone. Groundwater supply and climate change management by means of global atmospheric datasets. Preliminary results. Procedia Eng., 186:420427, 2017.

[3] FAO/UNESCO. Digital soil map of the world, 2013.

[4] M. Nucci. Water table monitoring in the Umbria region by ARPA Umbria. ARPA Umbria Report http://www.arpa.umbria.it/articoli/livelli-di-falda, in Italian, 2017.

[5] M. van Genuchten. A closed-form equation for predicting the hydraulic conductivity of unsaturated soils. Soil Sci. Soc. Amer. J., 44:892-898, 1980. 